\title{
Weak Fuzzy Equivalence and Equality Relations
}

\author{
Branimir Šešelja and Andreja Tepavčević* \\ Department of Mathematics and Informatics, University of Novi Sad, Serbia
}

\begin{abstract}
Weak fuzzy (lattice valued) equivalences and equalities are introduced by weakening the reflexivity property. Every weak fuzzy equivalence relation on a set determines a fuzzy set on the same domain. In addition, its cut relations are crisp equivalences on the corresponding cut subsets. Analogue properties of weak fuzzy equalities are presented. As an application, fuzzy weak congruence relations and fuzzy identities on algebraic structures are investigated.
\end{abstract}

AMS Mathematics Subject Classification (2000): primary 03B52, 03E72; secondary 06A15.

Keywords and phrases: lattice-valued fuzzy set, lattice-valued fuzzy relation, block, cut, fuzzy equivalence, fuzzy equality, fuzzy identity.

\section{Introduction}

Fuzzy equivalence relations belong to the most important notions of fuzzy structures. These were investigated from the beginning of fuzzy era. Instead of citing numerous relevant papers we refer to the book of Bělohlávek [2, in which an extensive list of references is provided. The notion of fuzzy equality was introduced by Höhle ([1] $)$ and then used by many others. In several papers, see e.g. 778], Demirci considers particular algebraic structures equipped with fuzzy equality relation, see also 4]. Bělohlávek (see [2, references there, and recent paper with Vychodil [3]) introduces and investigates algebras with fuzzy equalities.

Our approach in the present paper is cutworthy in the sense that cut substructures preserve crisp fuzzified properties. Due to this reason, the co-domain of our structures and relations is a fixed complete lattice, without additional operations. Differences of our approach and the foregoing mentioned ones is commented in the concluding section.

We introduce the notion of weak equivalence relation by weakening the reflexivity property. In this way, we obtain relations which determine fuzzy subsets on the same domain. In addition, the cut relations are precisely fuzzy equivalences on the cuts of the mentioned fuzzy set. In the case of weak fuzzy equality, these cuts are crisp equalities on the corresponding cut subsets.

\footnotetext{
* The research supported by Serbian Ministry of Science and Technology, Grant No. 144011 and by the Provincial Secretariat for Sci. and Techn. Development, Autonomous Province of Vojvodina, Grant "Lattice methods and applications".
} 
Our applications are situated in fuzzy universal algebra. We define a notion of fuzzy weak congruence relation on an algebra. It is a fuzzy weak equivalence which fulfills a substitution property with operations. Then, fuzzy subsets are fuzzy subalgebras and cut relations are crisp congruence relations on these subalgebras. Applying the obtained results, we have got some new results on fuzzy identities.

Although our research is mostly theoretical, we see its importance in real life applications. It is well known that fuzzy equivalences better model classification of objects then crisp relations. In case of weak equivalences, these applications, particulary in pattern recognition, can be even more suitable. This would be the task of our future investigation.

\section{Preliminaries}

We present basic notions related to fuzzy (lattice valued) structures and relations.

Let $(L, \wedge, \vee, \leq)$ be a complete lattice (for the notions from lattice and order theory see e.g. [6]).

A fuzzy set $\mu$ on a nonempty set $A$ is a function $\mu: A \rightarrow L$. For $p \in L$, a cut set, or a $p$-cut (sometimes simply a cut) of $\mu$ is a subset $\mu_{p}$ of $A$ which is the inverse image of the principal ideal in $L$, generated by $p$ :

$$
\mu_{p}=\{x \in A \mid \mu(x) \geq p\} .
$$

Other notions from the basics of fuzzy sets that we use are either defined in the sequel where they appear, or they can be found in any introductory text about fuzziness.

An $L$-valued relation $R$ on $A$ is

reflexive if $R(x, x)=1$, for every $x \in A$;

symmetric: $R(x, y)=R(y, x)$, for all $x, y \in A$;

transitive: $R(x, y) \wedge R(y, z) \leq R(x, z)$, for all $x, y, z \in A$.

An $L$-valued relation $R$ on $A$ is a lattice valued ( $L$-valued) equivalence relation on $X$ if it is reflexive, symmetric and transitive. An $L$-valued equivalence relation $R$ on $A$ is an $L$-valued equality relation if it fulfills the following

$$
R(x, y)=1 \text { if and only if } x=y .
$$

Throughout the paper, $L$ is supposed to be a fixed complete lattice.

\section{Lattice Valued Weak Equivalence Relations}

An $L$-valued relation $R$ on $A$ is weakly reflexive if for all $x, y \in A$,

$$
R(x, x) \geq R(x, y) \text {. }
$$

An $L$-valued relation $R$ on $A$ is a weak $L$-valued equivalence relation on $A$ if it is weakly reflexive, symmetric and transitive. In particular a weak $L$-valued equality $R$ on $A$ is an $L$-valued equivalence which fulfills also condition

$$
\text { if } u \neq v \text {, then } R(u, v)<\bigwedge_{x \in A} R(x, x) \text {. }
$$


In the following we demonstrate that each weak $L$-valued equivalence relation on a set determines a fuzzy subset on the same domain and we investigate the connection between the corresponding cut relations and cut subsets.

Theorem 1. If $R$ is a weak $L$-valued equivalence relation on a set $A$, then the mapping $\mu[R]: A \rightarrow L$, defined by

$$
\mu[R](x):=R(x, x)
$$

is an $L$-valued subset of $A$. In addition, for every $p \in L$, the cut relation $R_{p}$ is a crisp equivalence relation on the cut subset $\mu[R]_{p}$.

Proof. $\mu[R](x)$ is an $L$-valued subset of $A$ by the definition. Let $p \in L . x \in \mu[R]_{p}$ if and only if $R(x, x) \geq p$, and $(x, x) \in R_{p}$. Hence, $R_{p}$ is reflexive. Symmetry and transitivity of the cut $R_{p}$ follow directly from the symmetry and transitivity of fuzzy relation $R$.

Particular case of the above claim is obtained for fuzzy weak equalities.

Theorem 2. If $R: A^{2} \rightarrow L$ is a lattice valued weak equality on a set $A$, then for every $p \geq \bigwedge_{x \in A} R(x, x)$, the cut relation $R_{p}$ is a crisp equality on the cut subset $\mu[R]_{p}$.

Proof. Let $p \geq \bigwedge_{x \in A} R(x, x)$ and $x \in \mu[R]_{p}$. Then, $R(x, x) \geq p$ and $(x, x) \in R_{p}$. Let $(x, y) \in R_{p}$. Then $R(x, y) \geq p$. By the definition of weak $L$-valued equality, if $x \neq y$, then $R(x, y)<\bigwedge_{x \in A} R(x, x)$, hence, $x=y$, i.e., $R_{p}$ is a crisp equality on $\mu[R]_{p}$.

\section{Application in Algebra}

\subsection{L-Valued Subalgebras}

We advance some notions from fuzzy universal algebra, together with their relevant properties; more about crisp analogous properties can be found e.g., in the book [5].

Let $\mathcal{A}=(A, F)$ be an algebra and $L$ a complete lattice. As it is known, an $L$-valued (fuzzy) subalgebra of $\mathcal{A}$ is any mapping $\mu: A \rightarrow L$ fulfilling the following:

For any operation $f$ from $F, f: A^{n} \rightarrow A, n \in \mathbf{N}$, and all $x_{1}, \ldots, x_{n} \in A$, we have that

$$
\bigwedge_{i=1}^{n} \mu\left(x_{i}\right) \leq \mu\left(f\left(x_{1}, \ldots, x_{n}\right)\right) .
$$

For a nullary operation (constant) $c \in F$, we require that $\mu(c)=1$, where 1 is the top element in $L$.

Next, let $R: A^{2} \rightarrow L$ be an $L$-valued relation on $A$ (underlying set of the algebra $\mathcal{A})$. 
$R$ is said to be compatible with operations on $\mathcal{A}$ if for any ( $n$-ary) $f \in F$ and all $x_{1}, \ldots, x_{n}, y_{1}, \ldots, y_{n} \in A$, we have that

$$
\bigwedge_{i=1}^{n} R\left(x_{i}, y_{i}\right) \leq R\left(f\left(x_{1}, \ldots, x_{n}\right), f\left(y_{1}, \ldots, y_{n}\right)\right) \text {. }
$$

Let $E: A^{2} \rightarrow L$ be a fuzzy equality relation on $A$, which is compatible with operations on $\mathcal{A}$. We call $E$ a compatible $L$-valued equality on $\mathcal{A}$. Analogously, we define a weak compatible $L$-valued equality on $\mathcal{A}$ as a weak $L$-valued equality on $A$, which is also compatible with operations on $\mathcal{A}$.

The following facts about cuts of fuzzy subalgebras and of compatible fuzzy equalities are known.

Theorem 3. Let $\mathcal{A}$ be an algebra, $L$ a complete lattice, $\mu: A \rightarrow L$ a fuzzy subalgebra of $\mathcal{A}$, and $E$ a compatible fuzzy equality on $\mathcal{A}$. Then for every $p \in L$,

(i) the cut $\mu_{p}$ of $\mu$ is a subuniverse of $\mathcal{A}$, and

(ii) the cut $E_{p}$ of $E$ is a congruence relation on $\mathcal{A}$.

\subsection{Weak Lattice Valued Congruences}

Theorem 4. If $R: A^{2} \rightarrow L$ is a lattice valued weak congruence on an algebra $\mathcal{A}$, then the mapping $\mu[R]: A \rightarrow L$, defined by

$$
\mu[R](x):=R(x, x)
$$

is an $L$-valued subalgebra of $\mathcal{A}$.

Proof. Let $\mathcal{A}=(A, F)$ be an algebra and $f$ an n-ary operation from $F, f$ : $A^{n} \rightarrow A, n \in \mathbf{N}$ and let $x_{1}, \ldots, x_{n} \in A$.

Then

$$
\begin{gathered}
\bigwedge_{i=1}^{n} \mu[R]\left(x_{i}\right)=\bigwedge_{i=1}^{n} R\left(x_{i}, x_{i}\right) \leq R\left(f\left(x_{1}, \ldots, x_{n}\right), f\left(x_{1}, \ldots, x_{n}\right)\right)= \\
=\mu[R]\left(f\left(x_{1}, \ldots, x_{n}\right)\right) .
\end{gathered}
$$

Theorem 5. If $R: A^{2} \rightarrow L$ is a lattice valued weak congruence on $\mathcal{A}$, then for every $p \in L$, the cut relation $R_{p}$ is a congruence relation on the cut subalgebra $\mu[R]_{p}$.

Proof. Let $p \in L$. It is known from already mentioned facts that the cut relation $R_{p}$ is symmetric, transitive and compatible. To prove reflexivity, suppose that $x \in \mu[R]_{p}$. Then $R(x, x) \geq p$ and $(x, x) \in R_{p}$.

As a straightforward consequence of Theorem 2. we present the following property of lattice valued compatible weak equalities.

Corollary 1. Let $R: A^{2} \rightarrow L$ be a lattice valued compatible weak equality on $\mathcal{A}$ and for $x \in A$, let $R(x, x)=p$. Then $R_{p}$ is an equality relation on the cut subalgebra $\mu[R]_{p}$. 


\subsection{Fuzzy Identities}

If $E$ is a compatible $L$-valued equality on an algebra $\mathcal{A}$, and $t_{1}, t_{2}$ are terms in the language of $\mathcal{A}$, we consider the expression $E\left(t_{1}, t_{2}\right)$ as a fuzzy identity with respect to $E$, or (briefly) fuzzy identity. Suppose that $x_{1}, \ldots, x_{n}$ are variables appearing in terms $t_{1}, t_{2}$. We say that a fuzzy subalgebra $\mu$ of $\mathcal{A}$ satisfies a fuzzy identity $E\left(t_{1}, t_{2}\right)$ if for all $x_{1}, \ldots, x_{n} \in A$

$$
\bigwedge_{i=1}^{n} \mu\left(x_{i}\right) \leq E\left(t_{1}, t_{2}\right)
$$

In the present investigation we additionally consider the case in which the relation $E$ appearing in formula (1) is a weak compatible $L$-valued equality on $\mathcal{A}$. Then also we say that $\mu$ satisfies the fuzzy identity $E\left(t_{1}, t_{2}\right)$.

Proposition 1. 15] Let $\mathcal{A}$ be an algebra satisfying a (crisp) identity $t_{1}=t_{2}$ whose variables are $x_{1}, \ldots, x_{n}$. Let also $L$ be a complete lattice, $\mu: A \rightarrow L$ a fuzzy subalgebra of $\mathcal{A}$, and $E$ a compatible fuzzy equality on $\mathcal{A}$. Then, any fuzzy subalgebra $\mu: A \rightarrow L$ satisfies fuzzy identity $E\left(t_{1}, t_{2}\right)$.

Theorem 6. 15] Let $\mathcal{A}$ be an algebra, $L$ a complete lattice, $\mu: A \rightarrow L$ a fuzzy subalgebra of $\mathcal{A}$, and $E$ a compatible fuzzy equality on $\mathcal{A}$. Let also $\mu$ satisfies a fuzzy identity $E\left(t_{1}, t_{2}\right)$ in the sense of formula (1). Then for every $p \in L$, if $\mu_{p}$ is not empty then the crisp quotient algebra $\mu_{p} / E_{p}\left(\mu_{p}\right)$ satisfies the (crisp) identity $t_{1}=t_{2}$.

If we apply the above results to weak compatible fuzzy equalities, then we get that cut subalgebras (and not quotient algebras as above) fulfill the corresponding crisp identities, as demonstrated by the following theorem.

Theorem 7. Let $\mathcal{A}$ be an algebra, $L$ a complete lattice, $\mu: A \rightarrow L$ a fuzzy subalgebra of $\mathcal{A}$, and $E$ a weak compatible fuzzy equality on $\mathcal{A}$. Let also $\mu$ satisfies a fuzzy identity $E\left(t_{1}, t_{2}\right)$ in the sense of formula (11). Then for every $p \geq \bigwedge_{x \in A} E(x, x)$, if $\mu_{p}$ is not empty then the crisp subalgebra $\mu_{p}$ of $\mathcal{A}$ satisfies the (crisp) identity $t_{1}=t_{2}$.

Proof. Let $p \geq \bigwedge_{x \in A} E(x, x)$. Let $x_{1}, \ldots, x_{n}$ be elements from $\mu_{p}$. Then, $\mu\left(x_{1}\right) \geq$ $p, \ldots, \mu\left(x_{n}\right) \geq p$. Hence,

$$
p \leq \bigwedge_{i=1}^{n} \mu\left(x_{i}\right) \leq E\left(t_{1}\left(x_{1}, \ldots, x_{n}\right), t_{2}\left(x_{1}, \ldots, x_{n}\right)\right),
$$

since $\mu$ satisfies fuzzy identity $E$. On the other hand, by the definition of weak compatible fuzzy equality, we have that if $t_{1}\left(x_{1}, \ldots, x_{2}\right) \neq t_{2}\left(x_{1}, \ldots, x_{n}\right)$, then

$$
E\left(t_{1}\left(x_{1}, \ldots, x_{n}\right), t_{2}\left(x_{1}, \ldots, x_{n}\right)\right)<\bigwedge_{x \in A} E(x, x) \leq p,
$$

which is a contradiction with the fact that $E\left(t_{1}\left(x_{1}, \ldots, x_{n}\right), t_{2}\left(x_{1}, \ldots, x_{n}\right)\right) \geq p$. Hence, $t_{1}\left(x_{1}, \ldots, x_{2}\right)=t_{2}\left(x_{1}, \ldots, x_{n}\right)$, and $\mu_{p}$ satisfies the identity $t_{1}=t_{2}$. 


\section{Conclusions}

The paper deals with weak fuzzy equivalences on a set and with applications in algebra. From the algebraic aspect, our approach differs from the one developed in 3] (see also 2]). First, due to our cutworthy framework, we use lattice theoretic operations, and not additional ones (existing in residuated lattices). Next, we start with a crisp algebra and use a fuzzy equality to introduce fuzzy identities. In addition, our fuzzy equality is weakly reflexive, which, due to compatibility, enables determination of fuzzy subalgebras by its diagonal.

Our next task is to introduce and investigate the corresponding (weak) fuzzy partitions. Apart from algebraic application, these could be used in pattern recognition. Indeed, in addition to crisp and fuzzy partitions, weak partitions could model not only properties of whole domains, but also their fuzzy sub-domains (observe that a weak fuzzy equivalence possesses the fuzzy diagonal instead of the constant).

\section{References}

1. De Baets, B., Mesiar, R.: T-partitions. Fuzzy Sets and Systems 97, 211-223 (1998)

2. Bělohlávek, R.: Fuzzy Relational Systems: Foundations and Principles. Kluwer Academic/Plenum Publishers, New York (2002)

3. Bělohlávek, R., Vychodil, V.: Algebras with fuzzy equalities. Fuzzy Sets and Systems 157, 161-201 (2006)

4. Bodenhofer, U., Demirci, M.: Strict Fuzzy Orderings with a given Context of Similarity. Internat. J. Uncertain. Fuzziness Knowledge-Based Systems 16(2), 147-178 (2008)

5. Burris, S., Sankappanavar, H.P.: A Course in Universal Algebra. Springer, Heidelberg (1981)

6. Davey, B.A., Priestley, H.A.: Introduction to Lattices and Order. Cambridge University Press, Cambridge (1992)

7. Demirci, M.: Vague Groups. J. Math. Anal. Appl. 230, 142-156 (1999)

8. Demirci, M.: Foundations of fuzzy functions and vague algebra based on manyvalued equivalence relations part I, part II and part III. Int. J. General Systems 32(3), 123-155, 157-175, 177-201 (2003)

9. Di Nola, A., Gerla, G.: Lattice valued algebras. Stochastica 11, 137-150 (1987)

10. Goguen, J.A.: L-fuzzy Sets. J. Math. Anal. Appl. 18, 145-174 (1967)

11. Höhle, U.: Quotients with respect to similarity relations. Fuzzy Sets and Systems 27, 31-44 (1988)

12. Malik, J.N., Mordeson, D.S., Kuroki, N.: Fuzzy Semigroups. Springer, Heidelberg (2003)

13. Montes, S., Couso, I., Gil, P.: Fuzzy $\delta-\varepsilon$-partition. Information Sciences 152, 267-285 (2003)

14. Murali, V.: Fuzzy equivalence relations. Fuzzy Sets and Systems 30, 155-163 (1989)

15. Šešelja, B., Tepavčević, A.: Fuzzy identities. In: Proceedings of FUZZ-IEEE 2009, pp. 1660-1663 (2009)

16. Šešelja, B., Tepavčević, A.: On Generalizations of Fuzzy Algebras and Congruences. Fuzzy Sets and Systems 65, 85-94 (1994)

17. Šešelja, B., Tepavčević, A.: Fuzzy groups and collections of subgroups. Fuzzy Sets and Systems 83, 85-91 (1996)

18. Tepavčević, A., Vujić, A.: On an application of fuzzy relations in biogeography. Information Sciences 89(1-2), 77-94 (1996) 